\title{
Kurzfassung und Schlussfolgerungen
}

\author{
Carl Friedrich Gethmann, Peter Buxmann, Julia Distelrath, \\ Bernhard G. Humm, Stephan Lingner, Verena Nitsch, Jan C. Schmidt \\ und Indra Spiecker genannt Döhmann
}

\subsection{Zentrale Ergebnisse}

Die Digitalisierung von Arbeitswelten erreicht alle Bereiche öffentlich oder privat finanzierter Forschung und Entwicklung. Insbesondere lassen neuartige Ent-

C. F. Gethmann

Universität Siegen, Siegen, Deutschland

E-Mail: carl.gethmann@uni-siegen.de

P. Buxmann

Fachgebiet Wirtschaftsinformatik, Technische Universität Darmstadt, Darmstadt, Deutschland E-Mail: buxmann@is.tu-darmstadt.de

J. Distelrath

Schalkenbach, Deutschland

E-Mail: julia.distelrath@gmx.de

B. G. Humm

Hochschule Darmstadt, Darmstadt, Deutschland

E-Mail: bernhard.humm@h-da.de

S. Lingner $(\bowtie)$

Institut für qualifizierende Innovationsforschung und -beratung Gmbh (IQIB), Bad NeuenahrAhrweiler, Deutschland

E-Mail: Stephan.Lingner@iqib.de

V. Nitsch

Institut für Arbeitswissenschaft, RWTH Aachen, Aachen, Deutschland

E-Mail: v.nitsch@iaw.rwth-aachen.de

J. C. Schmidt

Department of Social SciencesHochschule Darmstadt, Darmstadt, Deutschland

E-Mail: Jan.Schmidt@h-da.de

I. Spiecker genannt Döhmann

Goethe Universität Frankfurt a.M., Frankfurt am Main, Deutschland

E-Mail: spiecker@jur.uni-frankfurt.de

(C) Der/die Autor(en) 2022

C. F. Gethmann et al., Künstliche Intelligenz in der Forschung, Ethics of Science and Technology Assessment 48, https://doi.org/10.1007/978-3-662-63449-3_7 
wicklungen der künstlichen Intelligenz große Veränderungen im Alltag grundlagenund anwendungsorientierter Forschung erwarten. Dennoch ist wissenschaftliche Forschung auch im Zeitalter von KI, Machine Learning und Big Data weiterhin auf den Menschen als Wissenschaftler/Wissenschaftlerin und Forscher/Forscherin angewiesen. Der Mensch bleibt daher als handelnder Akteur im Zentrum von Wissenschaft und Forschung. Ein etwaiger Ersatz des Menschen durch „starke KI“ ist dagegen nicht zu erwarten, da diese zumindest auf absehbare Zeit nicht erreichbar erscheint. KI-Systeme können daher zunächst eher als hilfreiche Werkzeuge in der Forschungspraxis verstanden werden: Auf den ersten Blick scheinen sie sich an technische Inventionen der Vergangenheit anzuschließen, wie die des Mikroskops oder des Fernrohrs, welche die wissenschaftliche Forschung und Erkenntnis bereits fundamental verändert haben. Ähnliches könnte auch für KI-getriebene Forschung erwartet werden. Allerdings führt die Selbstorganisationsfähigkeit von KI-Systemen im Modus des maschinellen Lernens (ML) zu einer Intransparenz von Forschungsprozessen, die eine Kontrolle, Validierung und Anerkennung abgeleiteter Aussagen, Modelle und Theorien erschweren oder gar unmöglich machen. Insofern kann der Wandel in der KI-getriebenen Forschung in diesem Modus sogar als disruptiv betrachtet werden. Ein allzu einfaches Werkzeug- und Instrumentenverständnis von KI führt hier also in die Irre; vielmehr sind ML-basierte KI-Systeme aufgrund ihrer verborgenen Dynamik und ihrer geringen Kontrollierbarkeit gewissermaßen als Instrumente zweiter Ordnung zu werten.

Für einen Wandel durch den Einsatz von KI-Systemen in Wissenschaft und Gesellschaft sprechen nicht nur Effizienzgewinne (im Sinne von mehr, schneller, billiger); sie stellen auch Ermöglichungs- und Infrastrukturtechniken dar, die qualitativ neue Zugänge zu Forschungsobjekten und -leistungen z. B. durch hohe Datenverfügbarkeit, Rechen- und sensorische Kapazitäten sowie durch schnelle Tools (semantic mining etc. ...) erlauben. Dadurch, dass sie sich oft im Nachhinein als „multi-purpose tools“ erweisen, sind produktive, aber oft auch zweischneidige und nicht-vorhersehbare Zwecksetzungen und Nutzungskontexte mit großer Tragweite für die Anwenderseite möglich. KI führt so in neue, auch disruptive Nutzungskontexte. Insofern dringt KI sogar in die Zweckdimension forschenden Handelns ein, in dem sie neue Zwecksetzungen ,,absichtslos“ induziert.

Was sich nun auf Seiten der wissenschaftlichen Erkenntnisbildung (disruptiv) ändert bzw. abzeichnet, ist ein Rückgang der auf Kausalität beruhenden Erklärungsleistung KI-vermittelter Forschung hin zu einem Forschungsmodus, der auf die Erkennung von Zusammenhängen gerichtet ist, sowie auf daraus abgeleitete Voraussagen. Dieser Modus kann zwar die Generierung neuer wissenschaftlicher Hypothesen unterstützen, ist aber selbst weniger theorieabhängig. Damit nimmt die Relevanz von Experimenten für die Widerlegung (Falsifikation) oder Bestätigung wissenschaftlicher Hypothesen ab; indirekte Formen der Falsifikation und Fragen der Kohärenz von Erklärungen spielen dann eine stärkere Rolle. Die o. g. Intransparenz von Forschung im Modus des maschinellen Lernens erschwert zusätzlich die Nachvollziehbarkeit von Forschungsprozessen und -ergebnissen. Im Detail sind hier auch etwaige Verzerrungen (Biases) in den Resultaten schwerer nachzuweisen und einzuordnen als mit herkömmlichen Forschungsmethoden. Es ist daher damit 
zu rechnen, dass mangels Überprüfbarkeit Vertrauensdefizite von entsprechenden KI-basierten Forschungsleistungen auftreten können, soweit ihnen nicht durch geeignete neuartige Validierungsverfahren entgegengewirkt werden kann.

Auf der anderen Seite wird die o. g. besondere Leistungsfähigkeit von KISystemen neue, bislang kaum zugängliche Forschungsfelder erschließbar machen, die insbesondere durch systemische Komplexität, Nichtlinearität und Instabilität ihrer Komponenten und Prozesse gekennzeichnet sind - und somit als schwach-kausal bezeichnet werden können. Weitergehend betrifft dies auch die Erweiterung bestehender Forschungsfelder und -gegenstände. Es handelt sich hierbei um Objekte und Systeme, die bislang als zu komplex galten, die aufgrund von hohem Energie-, Stoff- und Informationsaustausch mit ihrer Umwelt schwer zu isolieren waren und deren Dynamik insgesamt groß ist. Dies ist typisch für Biosysteme und Objekte der Lebenswissenschaften, aber auch für physikalische Systeme der Hochenergiephysik, der Kosmologie und Astronomie sowie der Festkörperphysik, ferner für technische bzw. soziotechnische Systeme.

Insgesamt stellen KI, Machine Learning und Big Data Katalysatoren der Transformation des forschenden Wissenschaftssystems in Richtung Anwendungsorientierung dar. Dabei zeigt sich ein Übergang von einem erkenntnis-, theorie- und erklärungsorientierten Regime der Forschung zu einem, das nutzenorientierter ist. Diese Transformation spiegelt sich auch in der öffentlichen und innerwissenschaftlichen Legitimationsrhetorik wissenschaftlichen Erkenntnishandelns wider. Allgemein ist diese Richtung von Forschung enger mit einer technik- und ingenieurwissenschaftlichen Wissenschaftshaltung verbunden als mit einer grundlagenorientierten. Sie ist stärker phänomenologisch und weniger fundamental ausgerichtet. Es geht ihr mehr um Technik als um Theorie, mehr um Werke als um Wahrheit, mehr um Gestaltung als um Erkennen. Dabei bleibt das erkenntnis-, theorie- und erklärungsorientierte Regime der Forschung weiterhin existent, es tritt nur gegenüber dem anwendungsund nutzenorientierten Regime in den Hintergrund. KI, Machine Learning und Big Data fördern somit den Übergang von „Science“ zu „Techno-Science“. In gewisser Hinsicht rückt dann Wissenschaft - in aller Ambivalenz - näher an die (nun verwissenschaftlichte) Gesellschaft heran.

In der Arbeitswelt der Forschenden führt der Trend der Digitalisierung und KI-gestützten Automatisierung zu moderaten Anpassungsanforderungen an die Forschenden. Erforderliche Kompetenzen und Fragen der Work-life Balance werden von den Betroffenen zumeist als wenig kritisch wahrgenommen. KI wird vielmehr als willkommene Unterstützung und Befreiung von administrativen Routineaufgaben im Arbeitsalltag gesehen. Als kritische Punkte für verwendete KI-Systeme werden dagegen von den Betroffenen genannt: unzureichende Standards und mangelnde Transparenz (Black-Box-Probleme, Biases, fehlende Reflexion), Missbrauch und Datenschutzprobleme sowie vereinzelt auch ethische Fragen (befürchtete Autonomieverluste und Technikabhängigkeit). Insgesamt steigt für die Forschenden die psychische und physische Belastung am Computerarbeitsplatz, sofern von Arbeitgeberseite nicht stärker auf eine menschengerechte Arbeits(platz) gestaltung geachtet wird. Für den deutschen Arbeitsmarkt könnte die KI-getriebene Automatisierung und die damit einhergehende Zunahme der Globalisierung von 
Wissensarbeit in der Forschung eine Abwanderung von entsprechenden Arbeitsplätzen in Niedriglohnländer nach sich ziehen.

Aus rechtswissenschaftlicher Sicht sieht sich KI-basierte Forschung insbesondere mit den Herausforderungen der Forschungsevaluation und des Datenschutzes konfrontiert. Der erste Punkt könnte in einen Konflikt zwischen KI und Big Data einerseits und fairer Forschungsevaluation andererseits münden. So würde KI-basierte Forschung durch ihre „Datenfixiertheit“ voraussichtlich neue und ggf. einseitige Benchmarks für die Evaluation von Forschungsleistungen nach sich ziehen, die Fachkulturen nicht-empirisch arbeitender Disziplinen benachteiligen könnten. Hier stellt sich die Frage, wie künftig für einen fairen Wettbewerb zwischen den verschiedenen wissenschaftlichen Disziplinen gesorgt werden kann und konkret, in welcher Weise entsprechende Evaluationsmethoden ggf. angepasst werden sollten, um den Besonderheiten der Fachkulturen weiterhin Rechnung zu tragen. Diese Position wird auch vom Wissenschaftsrat unterstützt (Wissenschaftsrat 2020).

Ein anderer Punkt betrifft den grundsätzlich bestehenden Konflikt zwischen Wissenschaftsfreiheit und Datenschutz: Dieser Konflikt wird sich angesichts des hohen Datenbedarfs KI-basierter Forschung einerseits und dem Gebot der Datensparsamkeit der Europäischen Datenschutz-Grundverordnung DSGVO andererseits voraussichtlich verschärfen - zumindest auf „sensiblen“ Forschungsfeldern. Zwar bietet die Zweckbindung aus der Verordnung eine mögliche Orientierung für die rechtmäßige Datenerhebung und -nutzung in der klassischen Forschung; sie wird aber z. B. dem explorativ-suchenden Modus von Big-Data-basierter KI für die Mustererkennung in unstrukturierten Datensätzen nicht gerecht. Für einen angemessenen Datenzugang in der KI-unterstützten Forschung bedarf es daher neuer Vorgaben zur besseren Ausgestaltung des Umgangs mit Forschungsdaten, die über das derzeit geltende Datenschutzrecht hinausgehen (vergl. auch Deutscher Ethikrat 2017). Hierzu sind geeignete Kriterien und Standards zu entwickeln und zu rechtfertigen.

Das Minimierungsgebot und die Zweckbindungserfordernis der DSGVO sind gleichwohl nicht grundsätzlich forschungsfeindlich, da die Verordnung Öffnungsklauseln vorsieht und in der Rechtsprechung Raum für wohlwollende Auslegungen zulässt. Dennoch wären klarere, forschungsfreundliche Regelungen wünschbar, die den Forschenden im Zeitalter von KI und Big Data Ungewissheiten und Auslegungsfragen rechtmäßiger Datenverwendung ersparen würden.

\subsection{Wünschbare Forschungsbedingungen im Zeitalter von KI}

\subsubsection{Strategievorschläge an das Wissenschaftssystem}

Die weitere Gestaltung des forschenden Wissenschaftssystems sollte sich an bewährten Qualitätsmaßen moderner Forschung orientieren. Dabei soll KI-basierte Forschung unter Bedingungen erfolgen, unter denen sie ihre wissenschaftlichen 
Geltungsansprüche hinreichend begründet, ihre Prämissen offenlegt und ihre Resultate im wissenschaftlichen und ggf. auch im weiteren gesellschaftlichen Diskurs validiert. Nur so kann ein intersubjektiver Status und folglich auch eine wissenschaftliche Robustheit von Ergebnissen KI-basierter Forschung erreicht und sichergestellt werden. Damit wissenschaftliche Ergebnisse nachvollziehbar sind, sollen die zugrunde liegenden Daten zugänglich sein. Des Weiteren sollen die eingesetzten Berechnungsverfahren benannt werden.

Hierzu sind zunächst vertrauen-schaffende Mechanismen zu implementieren, die die technisch-induzierte Intransparenz von KI-Systemen und ihrer Beiträge zu entsprechenden Forschungsleistungen weitestgehend reduzieren. Weiterhin sollten die den in der Forschung eingesetzten KI-Systemen zugrunde liegenden wissenschaftlichtechnischen Normen offengelegt werden, um z. B. mögliche technisch-bedingte Verzerrungen (Biases) von Forschungsergebnissen erkennbar zu machen. Schließlich sind auch flankierende Maßnahmen zur Offenlegung (und ggf. Legitimation) des jeweiligen Erkenntnisinteresses an KI-basierter Forschung zu ergreifen, um die Resultate in Wissenschaft und Gesellschaft besser einbetten zu können.

Entsprechende Maßnahmen sind möglichst frühzeitig zu implementieren, um die Forscher/Forscherinnen für epistemische Risiken, die mit der partiellen Intransparenz, Eigendynamik und Unkontrollierbarkeit von KI, insbesondere im Modus des Machine Learning verbunden sind, rechtzeitig zu sensibilisieren und in ihre wissenschaftlichen Interpretationen der Gesamtergebnisse einfließen zu lassen. So wird vermieden, dass sich im Umgang mit KI in der Forschung eine mögliche „Sorglosigkeit“ herausbildet und verfestigt. Den Forschenden muss bewusst werden, dass KI im Modus des maschinellen Lernens teilweise undurchschaubar ist und daher als Forschungsinstrument nur bedingt vertraut werden kann. Vor diesem Hintergrund sollte das Forschungsfeld der so genannten ,erklärbaren KI“ (engl. Explainable AI (XAI)) verstärkt gefördert werden, das darauf abzielt, die Intransparenz von KI-Systemen zumindest zu reduzieren und Nachvollziehbarkeit und Interpretierbarkeit zu ermöglichen. Die Forderung nach mehr Transparenz von KI-Prozessen wird auch in außerwissenschaftlichen Kontexten erhoben (Heesen et al. 2020).

Forschungsprojekte, in denen KI-Systeme eingesetzt werden, sollten daher auch stets personelle Informatik-Kompetenz vorhalten, um den wissenschaftlichen Risiken naiver KI-Verwendung zu begegnen. Darüber hinaus sind in den jeweiligen Forschungskontexten „Gütemaße“ ggf. einzusetzender KI-Systeme festzulegen, die den Umfang ihres jeweiligen Einsatzes für projektspezifische Aufgaben sinnvollerweise bestimmen bzw. begrenzen. Auch hierfür wird informationstechnische Beratungskompetenz benötigt.

Forscher/Forscherinnen sollten sich auch im Zeitalter von KI weiterhin als Urheber des Forschungshandelns begreifen. Sie bleiben trotz ,,autonomer“ informationstechnischer Teilprozesse treibende Kraft in der Forschung - nicht zuletzt wegen der besonderen Reflexionserfordernisse zuweilen intransparenter KI-basierter Verfahren und im Interesse zustimmungsfähiger Wissenschaft. 


\subsubsection{Empfehlungen für den Ausbildungs- und Weiterbildungsbereich}

Heutigen und zukünftigen Forschern/Forscherinnen sollten verpflichtende Aus- und Weiterbildungsangebote gemacht werden, die ihnen die notwendigen theoretischen und praktischen Grundkompetenzen KI-basierter Forschung inklusive entsprechender Erfahrungen und Fertigkeiten vermitteln. Hierfür sind frühzeitig entsprechende Curricula zu entwickeln oder zu ergänzen und ggf. um nicht mehr im Forschungsalltag benötigte Kompetenzen zu verschlanken.

Diese Bildungsmaßnahmen sollen nicht nur das technische und das praktische Verständnis über KI-Systeme in der Forschung stärken, sondern auch dazu beitragen, den „KI-Hype“ auf die Sachebene hin zu entmystifizieren. Sie sollen dabei die Reflexivität und Urteilsfähigkeit von Forschenden in neuartigen ,kollaborativen" KI-Umgebungen ermöglichen bzw. erhalten. Dabei sind neue wissenschaftliche Risiken aber auch neue erkenntnisbildende Chancen durch KI-basierte Forschung genauso zu vermitteln, wie auch herkömmliche Methoden, die im Zeitalter von KI mehr an Bedeutung gewinnen können (Statistik, Wahrscheinlichkeitstheorie etc.). Ferner sind die Forscher/Forscherinnen in diesem Zusammenhang weiterhin auf die bewährten wissenschaftskonstituierenden Normen, wie die Sicherung der Falsifizierbarkeit und Reproduzierbarkeit ihrer Forschungsresultate hin zu sensibilisieren und zu verpflichten.

\subsubsection{Wünsche an die Regulierung von KI-basierter Forschung}

Die Verfahren der Forschungsevaluation sollten auch im Zeitalter von KI und Big Data einen fairen Wettbewerb zwischen den unterschiedlichen Fachkulturen erlauben und sollten daher insbesondere wenig datengenerierende Disziplinen z. B. im Wettbewerb um öffentliche Fördermittel nicht benachteiligen. Hierzu sind wissenschaftliche Begutachtungsverfahren ggf. anzupassen oder zu ergänzen.

Umgekehrt sind für öffentlich finanzierte, datenintensive Forschungen klarere Regelungen der Europäischen Datenschutz-Grundverordnung wünschbar, die das Minimierungsgebot und die Zweckbindung der Datenverwendung eindeutiger und forschungsfreundlicher gestalten. Nur so lässt sich dem explorativen Forschungsmodus von KI in Verbindung mit Big Data gerecht werden.

\section{Literatur}

Deutscher Ethikrat (Hrsg) (2017) Big Data und Gesundheit - Datensouveränität als informationelle Freiheitsgestaltung. Stellungnahme. Deutscher Ethikrat, Berlin. https:/www.ethikrat.org/ fileadmin/Publikationen/Stellungnahmen/deutsch/stellungnahme-big-data-und-gesundheit. pdf. Zugegriffen am 20.11.2020 
Heesen J, Grunwald A, Matzner T, Roßnagel A (2020) Ethik-Briefing. Leitfaden für eine verantwortungsvolle Entwicklung und Anwendung von KI-Systemen. Whitepaper. München: Plattform Lernende Systeme. https://www.plattform-lernende-systeme.de/files/Downloads/ Publikationen/AG3_Whitepaper_EB_200831.pdf. Zugegriffen am 17.12.2020

Wissenschaftsrat (2020) Zum Wandel in den Wissenschaften durch datenintensive Forschung. Drs. 8667-20. Wissenschaftsrat, Köln. https://www.wissenschaftsrat.de/download/2020/8667-20. pdf?_blob=publicationFile\&v=5. Zugegriffen am 20.11.2020

Open Access Dieses Kapitel wird unter der Creative Commons Namensnennung 4.0 International Lizenz (http://creativecommons.org/licenses/by/4.0/deed.de) veröffentlicht, welche die Nutzung, Vervielfältigung, Bearbeitung, Verbreitung und Wiedergabe in jeglichem Medium und Format erlaubt, sofern Sie den/die ursprünglichen Autor(en) und die Quelle ordnungsgemäß nennen, einen Link zur Creative Commons Lizenz beifügen und angeben, ob Änderungen vorgenommen wurden.

Die in diesem Kapitel enthaltenen Bilder und sonstiges Drittmaterial unterliegen ebenfalls der genannten Creative Commons Lizenz, sofern sich aus der Abbildungslegende nichts anderes ergibt. Sofern das betreffende Material nicht unter der genannten Creative Commons Lizenz steht und die betreffende Handlung nicht nach gesetzlichen Vorschriften erlaubt ist, ist für die oben aufgeführten Weiterverwendungen des Materials die Einwilligung des jeweiligen Rechteinhabers einzuholen. 